Conclusion ESD is safe and effective in all ages. No significant difference was seen in complication rates between age groups and all adverse events were endoscopically treatable with no long term sequelae. We feel that age should not be a barrier to the use of ESD for early Barrett's neoplasia.

\section{PTU-047 POEM COMPARES FAVORABLY WITH LAPAROSCOPIC HELLERS MYOTOMY AS TREATMENT FOR ACHALASIA}

Sebastian Zeki*, Abraham Botha, Zoi Vrakopoulou, Lavinia Barbieri, Francesco Di Maggio, Jason Dunn, Terry Wong, Jafar Jafari. Guy's and St Thomas' Hospitals, London, UK

\subsection{6/gutjnl-2019-BSGAbstracts.260}

Introduction Achalasia is a rare disorder with several treatment options. Laparoscopic Heller's myotomy (LHM) has been the gold standard of treatment, however per-oral endoscopic myotomy (POEM) was recently established as a treatment option in achalasia proving itself an effective and safe procedure. The aim of this study was to summarize and compare the efficacy and safety of LHM and POEM for esophageal achalasia.

Materials and methods A prospective review was performed for all patients in our institution who have undergone LHM or POEM from 10/2012 to 06/2018, and have completed a minimum follow up period of 6 months. All patients underwent a standardized diagnostic work-up including gastroscopy, barium study and high-resolution manometry (HRM). All patients underwent a clinical evaluation 6 weeks after treatment and a surveillance gastroscopy in the 1st postoperative year, while further investigations were performed for patients with recurrent symptomatic. HRM and $\mathrm{pH}$ studies were offered to all patients after 6 months. Standardized questionnaires evaluating Eckardt, GERD and achalasia QoL (Urbach) scores were completed before and after each procedure. Statistical analyses were performed using SPSS version 23.0. Repeated measurements analysis of variance was used to compare changes in mean values during follow up and between the two types of surgery. $P$ values $<0.05$ were considered statistically significant.

Results Sample consisted of 80 patients (34 women and 46 men) with mean age 42.6 years (16.2). (There was a high rate of loss to follow-up due to distance from the facility). 39 of the sample underwent POEM and 41 underwent LHM. The two study groups were similar in terms of sex, age and type of achalasia $(p>0.05)$. LHM patients had greater follow up time, thus all analyses used time of follow up as a covariate. Qol score was significantly improved in both study groups $(p<0.001)$ and the degree of improvement was similar between the two groups $(p=0.486)$. Eckardt scores also showed a significant improvement in both groups, but repeated measurements analysis revealed a significant interaction effect with time $(p=0.038)$ indicating a greater improvement in POEM group. Furthermore, GERD score had a similar and significant decrease in both POEM and LHM group $(p=0.439)$. A subgroup analysis for those with and without prior treatments showed that Qol score had a similar mean difference among pre- and post measurements and between the two surgical groups. Moreover, Eckardt score change was similar between LHM and POEM patients without prior treatments $(p=0.724)$, but in those with prior treatments the improvement tended to be greater in POEM group $(p=0.087)$. Additionally, GERD score change was similar between LHM and POEM patients without prior treatments $(\mathrm{p}=0.979)$, but in those with prior treatments the improvement was greater in LHM group $(p=0.018)$. Postoperative Eckardt score $\leq 3$ was recorded in $87.2 \%$ of the patients of POEM group and in $78.4 \%$ of the patients of LHM group $(\mathrm{p}=0.308)$.

Conclusions Both LHM and POEM are efficient for the treatment of achalasia in terms of significant improvement in QoL, GERD and Eckardt scores. However, POEM tends to achieve greater improvement of the Eckardt score in patients with prior treatments, even if the difference is not statistically significant in this small sample $(\mathrm{p}=0.087)$. On the other hand, LHM reveals statistically significant difference regarding the decrease of GERD score in the same subgroup of patients $(\mathrm{p}<0.05)$. Finally, there was no significant difference regarding the complication rates for both groups (Clavien-Dindo I-IIIa).

\section{PTU-048 A COMPARATIVE QUALITATIVE SURVEY OF PATIENT EXPERIENCE IN BARRETT'S OESOPHAGUS}

1,3,5 James Britton*, ${ }^{5}$ Paraskevi Taxiarchi, ${ }^{5}$ Glen Martin, ${ }^{2}$ Robert Willert, ${ }^{4}$ Maria Horne, 3,5 Shaheen Hamdy, ${ }^{3,5}$ John McLaughlin, 3,5Yeng Ang. 'Wrightington, Wigan and Leigh NHS Trust, UK; ${ }^{2}$ Manchester University NHS Foundation Trust, UK; ${ }^{3}$ Salford Royal NHS Foundation Trust, UK; ${ }^{4}$ University of Leeds, UK; ${ }^{5}$ The University of Manchester, UK

Introduction

10.1136/gutjnl-2019-BSGAbstracts.261

This study aimed to assess Health Related Quality of Life (HRQoL) in patients with non-dysplastic Barrett's oesophagus (NDBO) and endoscopically treated dysplastic Barrett's oesophagus (DBO).

Methods This quantitative, self-administered questionnaire study was conducted across three NHS hospitals. Data was also collected from three other cohorts; GORD, colonic polyp surveillance and healthy individuals. HRQoL measurement included the Short Form-36 (SF-36), Gastrointestinal Symptom Rating Scale (GSRS), Hospital Anxiety and Depression Scale (HADS) and the Cancer Worry Scale (CWS). Fisher's exact and Spearman's rank correlation tests were used for analysis alongside propensity score matching to adjust for age, sex and comorbidities.

Results 797 participants responded to the survey (response rate 38\%), of which 687 were eligible for analysis (NDBO $\mathrm{n}=306$, DBO $\mathrm{n}=49$, GORD $\mathrm{n}=132$, Colonic polyps $\mathrm{n}=$ 152 and Healthy $\mathrm{n}=48$ ). Mean SF-36 scores were significantly lower (worse) in the NDBO cohort compared to healthy controls across all 8 domains and both physical and mental component summary scores $(\mathrm{p}=0.009-\mathrm{p}=<0.001)$. 53\% of NDBO participants reported significant cancer worry comparable to those treated for $\operatorname{DBO}(50 \%, \mathrm{p}=0.933)$ and those undergoing colonic polyp surveillance $(51 \%, p=0.355)$. Significantly less cancer specific worry was reported in GORD participants $(43.4 \%, \quad \mathrm{p}=0.01)$. NDBO participants reported anxiety in $15.8 \%(n=48)$ and depression in $8.6 \%$ of cases which was statistically comparable to the other disease cohorts but higher than the healthy cohort (anxiety 4\% $\mathrm{p}=0.001$ and depression $0 \% \mathrm{p}=0.006$ ). Moderate-severe heartburn or acid regurgitation was found in $11 \%$ and $10 \%$ respectively in the NDBO cohort. This was comparable to the DBO cohort 